\title{
Primary healthcare workers at risk during COVID-19: An analysis of infections in HIV service providers in five districts of South Africa
}

\author{
K Rees, ${ }^{1,2}$ MB BCh, FCPHM (SA); J L Dunlop, ${ }^{1,3}$ MB BCh, MSc (Med) Child Health; S Patel-Abrahams, ${ }^{1}$ RN, CNP; H Struthers, ${ }^{1,4}$ PhD; \\ J A McIntyre, ${ }^{1,5} \mathrm{MB} \mathrm{ChB}, \mathrm{FRCOG}$ \\ ${ }^{1}$ Anova Health Institute, South Africa \\ ${ }^{2}$ Department of Community Health, School of Public Health, Faculty of Health Sciences, University of the Witwatersrand, Johannesburg, \\ South Africa \\ ${ }^{3}$ Division of Community Paediatrics, School of Paediatrics, Faculty of Health Sciences, University of the Witwatersrand, Johannesburg, South Africa \\ ${ }^{4}$ Division of Infectious Diseases and HIV Medicine, Department of Medicine, Faculty of Health Sciences, University of Cape Town, South Africa \\ ${ }^{5}$ School of Public Health and Family Medicine, Faculty of Health Sciences, University of Cape Town, South Africa
}

Corresponding author: KRees (rees@anovahealth.co.za)

Background. Protecting healthcare workers (HCWs) from COVID-19 is a global priority. Anova Health Institute (Anova) is the PEPFAR (US President's Emergency Plan for AIDS Relief) District Support Partner for the Johannesburg, Cape Town, Sedibeng, Capricorn and Mopani districts in South Africa, operating in public sector primary healthcare facilities. At the time of the emergence of COVID-19, Anova employed close to 4000 people: $41 \%$ community health workers (CHWs), 23\% data staff, 20\% nurses and doctors, $12 \%$ management/ support and $5 \%$ allied HCWs.

Objectives. To describe rates of COVID-19 diagnosis in Anova-employed HCWs in five districts.

Methods. Employees exposed to, tested for or diagnosed with COVID-19 were required to report the event. These reports were compiled into a database to monitor the impact of COVID-19 on the workforce. We kept a timeline of key events occurring at national and district level, including Anova's policies and their implementation, that was used to describe organisational response. We described the number of confirmed cases, cumulative incidence rates and testing rates, broken down by district and job category. We estimated expected deaths and the effect on time off work.

Results. Of Anova employees, 14\% ( $n=562)$ were diagnosed with COVID-19 by the end of September 2020. Cumulative incidence was highest in Sedibeng (29\%) and lowest in Mopani (5\%). All HCWs experienced high incidences: data staff 17\%, allied HCWs 16\%, CHWs $14 \%$, nurses and doctors $13 \%$, and management/support $11 \%$. At the peak of the epidemic, for 5 weeks, $>5 \%$ of employees were unable to work owing to exposure or infection, significantly disrupting service delivery. The additional administrative burden on managers was substantial.

Conclusions. It is critical that all cadres of HCWs are protected in the workplace, including in primary care settings, where better structures are needed to perform risk assessments and conduct outbreak investigations. CHWs and data staff may be at higher risk owing to poor infrastructure, limited power to negotiate working conditions, and limited experience of infection prevention and control. Their working conditions must be improved to reduce their risk.

S Afr Med J 2021;111(4):309-314. https://doi.org/10.7196/SAMJ.2021.v111i4.15434

Protecting healthcare workers (HCWs) from COVID-19 is a global priority. The World Health Organization estimates that up to $14 \%$ of COVID-19 cases are in HCWs, ${ }^{[1]}$ and Amnesty International has reported $>7000 \mathrm{HCW}$ deaths worldwide. ${ }^{[2]}$ In South Africa (SA), in early August 2020, the Minister of Health announced that $>27000$ HCWs had been diagnosed with COVID-19, and 240 had died. ${ }^{[3]}$ COVID-19 is a compensable disease under the Compensation for Occupational Injuries and Diseases Act No. 130 of 1993, with healthcare delivery and support staff classified as high-risk exposure occupations. ${ }^{[4]}$ HCWs are known to be at higher risk for COVID19 than the general community, even when not directly caring for suspected COVID-19 cases. $^{[5]}$ A survey of SA HCWs carried out by the Human Sciences Research Council found that close to $80 \%$ felt at risk because of their profession, ${ }^{[6]}$ but despite this high perception of risk, many barriers exist to implementing infection prevention and control (IPC) guidelines. ${ }^{[7]}$ Understanding the risk that HCWs face is critical to improving protective measures.
Anova Health Institute (Anova) is a USAID (United States Agency for International Development)-funded PEPFAR (US President's Emergency Plan for AIDS Relief) District Support Partner (DSP). In 2020, Anova supported five SA districts: two metropolitan municipalities (Cape Town in Western Cape Province and Johannesburg in Gauteng Province) and three district municipalities (Capricorn and Mopani in Limpopo Province and Sedibeng in Gauteng). PEPFAR DSPs receive funding to support the government in the provision of HIV, tuberculosis (TB) and sexually transmitted infection care and treatment services, including both direct service delivery (HCWs employed by Anova to work in government health facilities, integrated into facility services, e.g. professional nurses whose primary task is to initiate patients onto antiretroviral therapy) and technical assistance (e.g. supporting the implementation of new guidelines, training and mentoring, and quality improvement).

At the time of the emergence of COVID-19, Anova had close to 4000 employees, many of whom spent the majority of their working 
time in public sector primary care health facilities across the five abovementioned districts. The Anova staff complement was made up of $\sim 1615$ CHWs, 779 nurses and doctors, 895 data staff (data capturers and managers, largely facility based), 179 allied HCWs (pharmacy staff, social workers and dieticians) and 473 management or support staff. Many of the management and support staff are trained health professionals and work in health facilities or visit health facilities frequently.

Anticipating a large number of COVID-19 infections, the SA National Department of Health $(\mathrm{NDoH})$ and PEPFAR negotiated adjustments to their existing agreement to allow DSPs to support certain COVID-19 activities. The first of these was to add household screening for COVID-19 to CHWs' activities. CHWs already employed by Anova to conduct HIV activities, as well as newly employed CHWs, were assigned to support the government-led household screening programme. When household screening was phased out, these CHWs returned to their previous activities.

Another major area of support was IPC and facility preparedness. Patient flow was altered to allow facilities to function more safely by separating patients with respiratory symptoms into designated areas. DSPs supported this process, including through procurement and technical assistance. Anova staff did not manage COVID-19 patients, although they did provide HIV services to people suspected of having COVID-19. They did not routinely perform diagnostic swabs on people with COVID-19 symptoms.

\section{Objectives}

To describe rates of COVID-19 diagnosis in Anova-employed HCWs in five districts, to improve our knowledge of how COVID-19 has impacted on all categories of primary care HCWs.

\section{Methods}

\section{Anova's organisational response}

The COVID-19 epidemic presented workplace challenges that were unique and unforeseen. High risk perception due to global media attention and lack of knowledge about critical factors such as mode of transmission meant that COVID-19 could not be managed in the same way as other workplace risks. A leadership committee was set up within Anova, consisting of the highest-level leaders and advisors. Each district had a clinically trained co-ordinator to manage COVID-19 exposures and cases. This role included support for COVID-19 cases, for example providing information, assisting with access to isolation facilities, facilitating access to counselling services, tracing contacts of COVID-19 cases, responding to known exposures by making decisions on the level of risk and quarantining when necessary, recording and monitoring known exposures and cases, organising COVID-19 testing for symptomatic or exposed employees, and working with managers to identify areas of noncompliance with protocols and improve on these. In the metropolitan districts, 12 additional doctors were needed for these functions.

Policies were implemented that required all employees exposed to, tested for or diagnosed with COVID-19 to report this to their co-ordinators. The report included job title, age, gender, date of diagnosis, details of exposure and/or symptoms, test result, and whether self-quarantine was possible. The details of these reports were captured into a database by the district co-ordinators. Each week, district databases were collated into one central database. Approval from the co-ordinators who maintained district-level data was required in order to access special leave, incentivising complete reporting. The integrity of the collated data was checked every month by comparing district-level and collated databases, and detailed weekly reports were disseminated, which were verified by co-ordinators.

Anova organised and sponsored testing through private laboratories for staff in the following circumstances:

- Symptoms suggestive of COVID-19. These were determined according to the National Institute for Communicable Diseases/ $\mathrm{NDoH}$ guideline for clinical management of suspected or confirmed COVID-19 disease, updated with each version of the guideline..$^{[8]}$ Staff were required to report any illness to a designated clinician, who requested that they present to a laboratory for testing if any symptoms fitting the definition of a suspected case were present. This applied to all employees, including those in quarantine.

- Day 8 of quarantine (later day 7). High-risk contacts were required to quarantine for 7 days, whereafter those who continued to be asymptomatic were requested to test for possible return to work, as outlined in the National Institute for Communicable Diseases/NDoH guideline on management of staff in healthcare and laboratory settings with COVID-19 illness and exposure. ${ }^{[9]}$

National IPC and personal protective equipment (PPE) guidelines were followed, with one notable exception: when high infection rates were noted in data staff, it was made a requirement for all staff working in health facilities to use surgical masks and visors. ${ }^{[10]}$

\section{Setting}

Table 1 describes the five districts, including COVID-19 cases per 100000 people in the general population, as reported by the provincial departments of health (DoHs). Nationally, Cape Town and Johannesburg were among the metropolitan municipalities most affected by COVID-19, with Cape Town being the first hotspot, with the earliest peak. Of the district municipalities, Sedibeng district experienced the highest case numbers, with a cumulative incidence

Table 1. Selected demographics and cumulative incidence of COVID-19 in the general population of the five districts analysed

\begin{tabular}{|c|c|c|c|c|c|c|}
\hline & Type of municipality ${ }^{\star}$ & Population, $N^{*}$ & $\begin{array}{l}\text { Population density } \\
\left(\text { persons } / \mathbf{k m}^{2}\right)^{\star}\end{array}$ & $\begin{array}{l}\text { Estimated } \\
\text { medical scheme } \\
\text { coverage, } \%^{\star}\end{array}$ & $\begin{array}{l}\text { Cases/100 } 000 \\
\text { population, } \\
\text { end June } 2020^{\dagger}\end{array}$ & $\begin{array}{l}\text { Cases/100 } 000 \\
\text { population, } \\
\text { end Sept } 2020^{\dagger}\end{array}$ \\
\hline Cape Town & Metropolitan & 4140565 & 1693 & 22.2 & 1295 & 1856 \\
\hline Johannesburg & Metropolitan & 5201673 & 3162 & 22.2 & 402 & 1686 \\
\hline Sedibeng & District & 984810 & 236 & 20.8 & 167 & 1512 \\
\hline Capricorn & District & 1338763 & 62 & 8.3 & 17 & 384 \\
\hline Mopani & District & 1225473 & 61 & 6.8 & 14 & 178 \\
\hline South Africa & & 57900000 & 47 & 15.4 & 306 & 1171 \\
\hline
\end{tabular}


in the general population close to that of Johannesburg by the end of September. At the time of writing, Capricorn and Mopani districts had experienced cumulative incidence rates 4 and 9 times lower than Johannesburg, respectively.

\section{Analysis}

Using the database of employee cases, we described the number of confirmed cases, cumulative incidence rates over the period April - September 2020, and testing rates. We calculated cumulative incidence because it allows comparison between different groups, and broke down estimates by district to investigate patterns in locations with different background incidence, and job category to investigate differing risk between these groups. We estimated expected deaths using age-specific mortality rates from the Western Cape DoH to account for the age structure of the workforce, since age is such an important risk factor for poor outcomes. We also examined the effect on the workforce, using the number of leave days taken, and the number of employees known to be in quarantine or isolation.

\section{Results}

\section{Description of confirmed cases}

The first case in an Anova employee was diagnosed on 7 April 2020. Over the next 6 months, by the end of September 2020, 562 individuals had been diagnosed with confirmed COVID-19. This was $\sim 14 \%$ of all staff employed by Anova at the time.

Age and gender distribution of diagnosed cases closely mapped to the age and gender distribution of Anova staff at a district level: $78 \%$ of cases were in female staff, and women make up $76 \%$ of the staff complement overall; $33 \%$ of cases were in individuals aged $<30$ years old, v. $30 \%$ of the staff overall; $39 \%$ were in 30 - 40 -year-olds, v. $43 \%$ of staff; $20 \%$ were in 40 - 49 -year-olds, v. $19 \%$ of staff; $7 \%$ were in 50 - 59-year-olds, v. $5 \%$ of staff; and $2 \%$ were in individuals aged $\geq 60$ years, v. $2 \%$ of staff. There were 10 cases in people aged $\geq 60$ years.

\section{Districts}

The highest number of new cases was in the week ending 3 July 2020, largely owing to the timing of the Johannesburg district programme peak (Fig. 1). The Cape Town programme diagnosed the highest number of new cases in the week ending 8 May, and new cases declined from July. Sedibeng district programme peaked from 3 July to 24 July. Sixty-five percent of cases $(n=368)$ were diagnosed during a 5 -week period (20 June - 24 July).
By the end of September, Sedibeng had the highest incidence at $29.1 \%$ (Fig. 2) (general population incidence $1.5 \%$ ), followed by Cape Town (24.0\%; general population incidence $1.7 \%$ ) and then Johannesburg (13.5\%; general population incidence $1.5 \%$ ). Capricorn and Mopani employees had lower incidences, at $9.2 \%$ and $5.1 \%$, respectively (general population incidence $0.3 \%$ and $0.1 \%)$.

\section{Job categories}

CHWs made up the highest proportion of confirmed infections, $40 \%$ of the total, with $14 \%$ of all CHWs diagnosed with COVID19 (Table 2). Data staff made up $27 \%$ of the cases, with the highest cumulative incidence of confirmed infection over the period April - September 2020 at $17 \%$.

This pattern did not change substantially over time, and throughout the study period the majority of confirmed infections were in CHWs and data staff. Towards the end of May, the CHWs' main activities shifted away from household COVID-19 screening back to their usual activities, including HIV testing and patient tracing in facility and community settings.

The group with the second-highest rate of infections was the allied HCWs, who are facility based and client facing. This group includes pharmacists, social workers and dieticians. Nursing staff and medical officers had a $13 \%$ rate of confirmed infections,

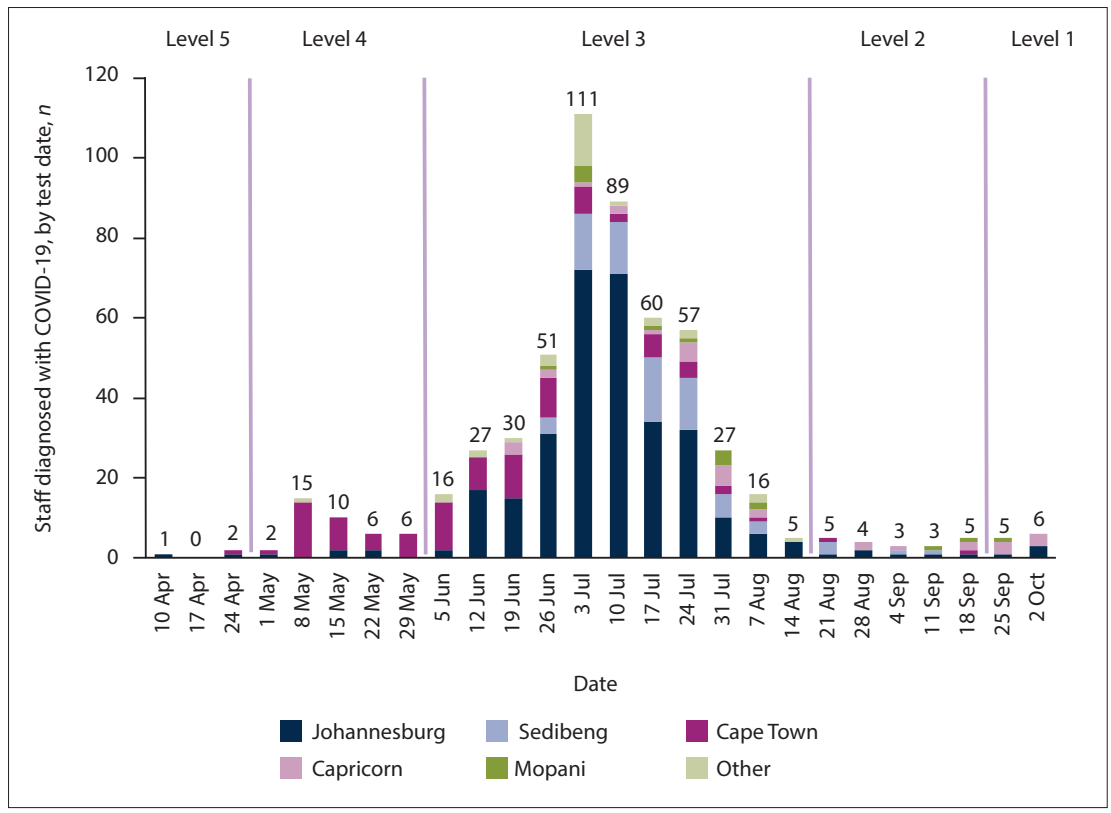

Fig. 1. Weekly new COVID-19 cases in Anova employees by test date by district, April - September 2020. (Other = head office employees, based in Johannesburg.)

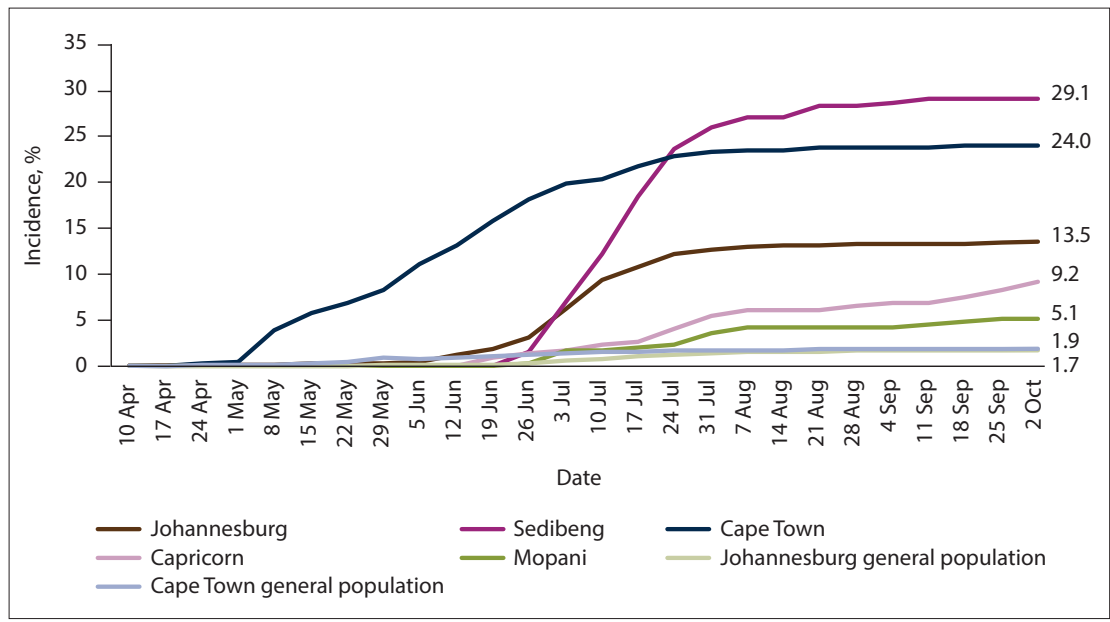

Fig. 2. Weekly COVID-19 cumulative incidence in Anova employees by test date by district, April September 2020. Data source for general population incidence: Gauteng Department of Health (https:// gpcoronavirus.co.za/) and Western Cape COVID-19 Dashboard (https://coronavirus.westerncape.gov. za/covid-19-dashboard). 
followed by management and support staff at $11 \%$. In comparison, $1.1 \%$ of the general population of SA had been diagnosed with COVID-19 by the end of September 2020.

\section{Hospitalisation and mortality}

Overall, 25 Anova employees were hospitalised ( $5 \%$ of cases), with 1 death $(0.2 \%$ of cases). Applying age-specific mortality rates reported in the Western Cape COVID-19 Dashboard to the age structure of Anova's cases, 9 deaths would be expected, or $1.6 \%$. The Western Cape's crude mortality rate was $3.8 \%$. At the end of September, there had been 1 COVID-19-related death among Anova staff, a crude rate of $0.2 \%$. The employee was a 37 -year-old male $\mathrm{CHW}$, who died in an intensive care unit.

\section{Testing patterns}

Over the study period, 1128 tests were conducted (in 1023 individuals), 238 (21\%) of which were positive ( $23 \%$ of individuals) Overall, $42 \%$ of cases were diagnosed through Anova-sponsored testing. Other ways in which staff accessed testing included through their personal medical providers and through DoH-instigated COVID-19 testing in facility staff. Access to other means of testing varied substantially by district and over time. In some cases, all staff at a facility were tested immediately following the identification of one positive test, and in some places universal testing was conducted as part of poorly defined HCW surveillance.

As community incidence declined, decreased positivity over time was evident. We also noted that a higher proportion of cases was diagnosed through Anovasponsored testing over time, which may point to decreased testing rates in the general population. A total of 1023 individuals equates to $25 \%$ of the staff having been tested. Assuming a similar positivity rate in staff tested through different mechanisms, the total would increase to 2676 staff members tested, or $68 \%$. In SA as a whole at the end of September, 3983533 individuals had been tested (7\%).

At the time of testing, 107 of the cases reported to Anova were reported to be asymptomatic (19\%).

\section{Effects on the workforce}

A total of 177 leave days were taken per 100 employees in July 2020, compared with 86 days per 100 employees in July 2019. A peak number of staff were in quarantine on 10 July ( $n=331$, or $8 \%$ of all staff) (Fig. 3 ). In addition, there were 209 active cases

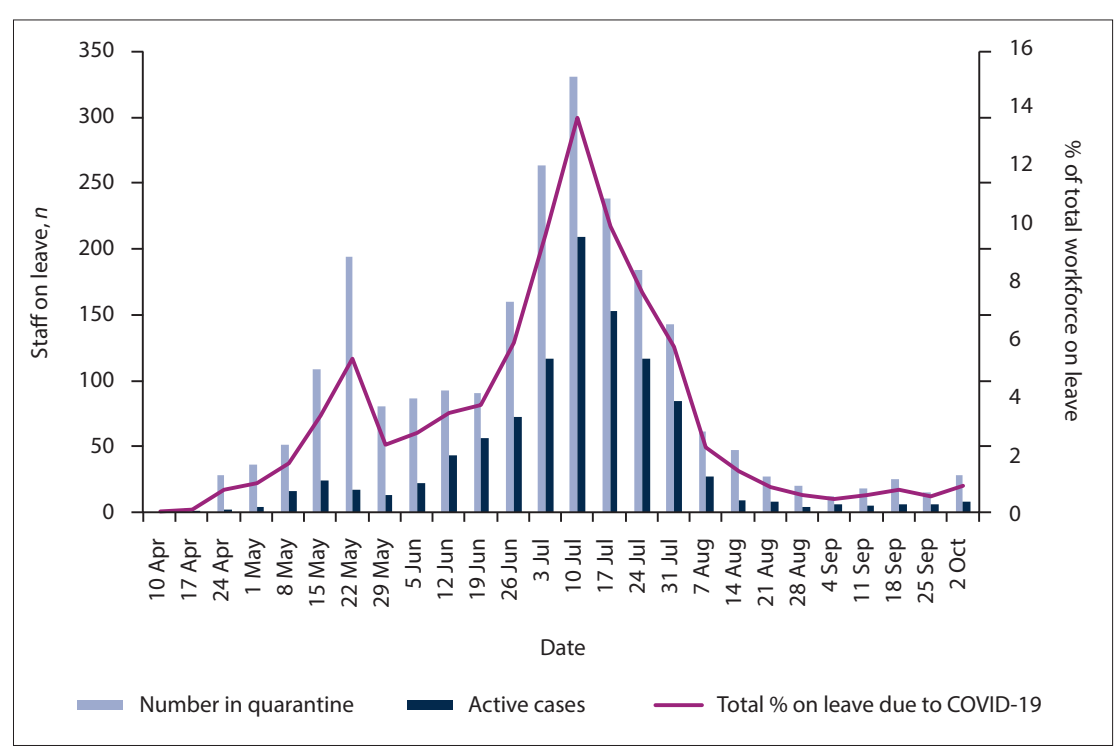

Fig. 3. Weekly number of active COVID-19 cases in Anova employees and employees in quarantine, April - September 2020. (defined as within 14 days of a positive test until 31 July, thereafter within 10 days of a positive test). This amounts to $14 \%$ of staff on leave due to COVID-19. More than 5\% of staff were on leave due to COVID-19 for a 5-week period, 26 June - 31 July 2020.

\section{Discussion}

We have described the cumulative incidence of COVID-19 in a group of HCWs at primary care level over a 6-month period that included the first COVID-19 peak in SA. We have shown that rates of infection were as high in frontline support staff as those in clinical HCWs. Although previous studies have compared rates in doctors and nurses, the impact on other groups of HCWs remains largely unknown. ${ }^{[1]]}$ In Anova employees, the cumulative incidence

\begin{tabular}{|c|c|c|c|c|c|c|}
\hline & Cases, $n$ & $\%$ of Anova cases & Total staff, $N$ & Rate, \% & Hospitalised, $n$ & Hospitalised, \% \\
\hline \multicolumn{7}{|l|}{ District } \\
\hline Cape Town & 99 & 18 & 412 & 24 & 8 & 8 \\
\hline Johannesburg & 311 & 55 & 2331 & 13 & 14 & 5 \\
\hline Sedibeng & 74 & 13 & 254 & 29 & 1 & 1 \\
\hline Capricorn & 32 & 6 & 349 & 9 & 0 & 0 \\
\hline Mopani & 16 & 3 & 312 & 5 & 0 & 0 \\
\hline Other & 30 & 5 & 283 & 11 & 2 & 7 \\
\hline \multicolumn{7}{|l|}{ Job category } \\
\hline CHWs & 224 & 40 & 1615 & 14 & 9 & 4 \\
\hline Data team & 154 & 27 & 895 & 17 & 3 & 2 \\
\hline Nursing and doctors & 102 & 18 & 779 & 13 & 10 & 10 \\
\hline Management and support & 54 & 10 & 473 & 11 & 2 & 4 \\
\hline Allied HCWs & 28 & 5 & 179 & 16 & 1 & 4 \\
\hline Total & 562 & 100 & 3941 & 14 & 25 & 5 \\
\hline
\end{tabular}


of COVID-19 exceeded that in the general population. Cumulative incidence was highest in data staff (17\%), followed by allied/other HCWs (16\%), CHWs (14\%) and nurses/doctors (13\%).

Although the higher observed incidence may be due to higher testing rates, in Anova employees testing coverage was 4 - 10 times that of the general population, and the cumulative incidence was 13 times higher overall, and more than 15 times higher in Cape Town and Sedibeng. In the mining industry, a testing rate of about 40 times that in the general population and an incidence four times higher has been observed. ${ }^{[12]}$ In a cohort in the UK and USA, frontline HCWs were 3.4 times more likely than the general population to test positive for COVID-19, after adjusting for testing patterns. ${ }^{[5]}$ It is likely that the higher incidence of COVID-19 infection in Anova employees was not solely because of increased testing, but rather due to exposure to unidentified people infected with COVID-19 who were seeking other health services, and transmission between healthcare workers in the workplace.

Despite the relatively high incidence, our observed mortality rate was lower than expected. Although our estimate of expected deaths is adjusted for age, it has not been adjusted for one of the most important known risk factors for poor outcomes - comorbidities. In the general population, people known to be high risk due to comorbidities were probably tested more often, which would not be the case in HCWs. It is also possible that Anova staff had fewer high-impact comorbidities, or that these were better controlled as a result of better access to healthcare (due to working within the system). Accommodations were made for staff members who had comorbidities (as well as those aged $\geq 60$ years), with many working remotely or adjusting their tasks to be less risky. In addition, increased testing in Anova employees due to guidelines specific to HCWs probably led to more asymptomatic and mild cases being diagnosed, compared with the general population.

Data staff and CHWs may be at high risk of being infected with COVID-19 for several reasons. Data capturers and many CHWs work in health facilities, usually in woefully inadequate rooms. Data capturers are a group of staff that has expanded over recent years as health information systems have become more central to service delivery, but facility infrastructure has not kept up with the increased space requirements. Data capturing rooms at primary care facilities are typically small and overcrowded with limited ventilation. CHWs frequently have no allocated space in health facilities and work in common areas such as tea rooms. This makes it difficult to control the environment. Both cadres have not been well integrated into the health system, with many being paid and managed externally by nongovernmental organisations. As such, they have very limited power to negotiate safer working conditions. Prior to this pandemic, data staff had not been trained in PPE use, and CHWs and data staff had very limited experience of PPE or IPC. In addition, their incomes are lower than professional staff, making it more likely that they travel long distances to work using public transport, and live in conditions that make social distancing challenging.

Despite many of the prevention measures for COVID-19 being the same as for TB, in general, IPC has been neglected in primary care spaces. ${ }^{[13]}$ Caring for COVID-19 patients is clearly a source of risk, but HCWs are also exposed to patients without clear COVID-19 symptoms who are seeking unrelated health services. Infrastructure is suboptimal at many facilities, with ever-growing populations, services provided, and numbers of staff needed to implement health programmes. Suboptimal infrastructure and lack of space makes social distancing difficult, and ventilation is frequently problematic. A study of KZN primary care sites found that half the rooms studied had inadequate ventilation, even with all windows and doors kept open. ${ }^{[13]}$ During winter, people are reluctant to open doors and windows, further hampering ventilation. With growing evidence for airborne transmission of SARS-CoV-2, it has become clear that ventilation is a key component of IPC. ${ }^{[14-16]}$ Engineering controls such as ventilation are more desirable than administrative controls and PPE, since they protect a greater number of people and do not rely on individual adherence. ${ }^{[17]}$ When infrastructure and ventilation are inadequate, administrative controls, such as separation of symptomatic patients, and PPE become even more important, including surgical masks for patients with respiratory symptoms. In this situation, cloth masks are inadequate for any HCW in a health facility.

In the primary care setting, the delineation between safe and unsafe areas is not always obvious. It became clear over the duration of the study that tea rooms and other shared spaces were frequently sources of high-risk contact between HCWs. Although staff were becoming used to PPE and other IPC measures, there was a dominant perception that patients would be the source of infection, although during outbreak investigations we found transmission between colleagues to be common. Similarly, in other healthcare settings, it has been found that direct patient-to-HCW contact is not likely to be the dominant source of transmission. ${ }^{[1,18]}$

During the emergence of COVID-19, the environment that healthcare organisations were working in changed rapidly. This was a time of great uncertainty in terms of policy and trying to predict how the epidemic would affect service delivery. With implementation of the national lockdown, health facilities across the country were focused on providing essential services only, to keep people out of facilities wherever possible. Alarming anecdotal reports suggested that the consequences of decreased service utilisation would be severe, ${ }^{[19]}$ and there is growing evidence that additional deaths could have been the result. ${ }^{[20]}$ Over time, the strategy shifted from encouraging fewer health facility visits to emphasising facility preparedness, which concentrated on IPC measures, including screening for COVID-19 symptoms, and separating symptomatic patients to demarcated areas. ${ }^{[21,22]}$ The period between the peaks in Cape Town and other affected districts allowed time for planning and benchmarking, and there was additional time to prepare facilities in other districts.

The administrative burden due to COVID-19 for managers and HR practitioners at all levels was considerable. It included investigating and documenting exposures, paperwork for exposures and infections, paperwork for Compensation for Injury and Occupational Diseases applications, and new leave processes. For health sector managers, the balance between time spent on administration and that spent on quality improvement and patient care is already unfavourable, and this additional burden worsened the situation. Within the structures specifically managing COVID-19 at Anova, the district co-ordinators could not manage the workload themselves, and 12 doctors in the organisation were needed to make decisions about exposures and testing, support monitoring and improve IPC procedures, further impacting on service delivery. The impact of disruptions, anxiety, fear and bereavement on individuals and the organisation was considerable.

It is difficult to explain the different rates of confirmed infection in Anova employees in different districts, as there are many potential contributing factors. These include varying approaches to contact tracing, containment and testing at health facilities from the different departments of health. Although Anova had standardised procedures, staff members were often tested according to district $\mathrm{DoH}$ procedures. Carefully planned and implemented facility preparedness protocols 
in primary care facilities in Johannesburg may have protected staff. The steep rise in cases and high cumulative incidence in Sedibeng employees is notable. Although Sedibeng is not a metropolitan district it is adjacent to Johannesburg, which may explain its delayed but high infection rates. It is possible that it took longer for community transmission to be established in this district, but once in the relatively small community, it was able to spread quickly.

\section{Study strengths and weaknesses}

We report on a large group of HCWs, including often-neglected cadres, through a period of high COVID-19 incidence in five districts. The range of HCWs and districts, and the consistency of symptom- and exposure-based surveillance, make this information valuable. Limitations include differing testing patterns in different areas, and at different times. Surveillance was symptom-based, and there was no regular testing of asymptomatic employees. Many asymptomatic infections were therefore probably not identified. Since Anova employees work in the public sector, an understanding of corresponding patterns in DoH HCWs would enhance this analysis. Data on comorbidities were not included because they were selfreported from employee to employer, and we think are unlikely to be complete. We also did not have access to socioeconomic data other than employment status and job title, and so were unable to report on this aspect in a more nuanced way. In addition, the CHWs employed by this relatively well-funded organisation may not be representative of CHWs across the country, who may be employed by organisations without our managerial and clinical capacity, which could make them even more vulnerable.

\section{Conclusions}

All HCWs, including data capturers, CHWs and administration staff working in health facilities, are at increased risk of contracting COVID-19. IPC and PPE guidelines need to reflect this. Increased attention needs to be paid to the health and safety of workers in the primary care setting, and efforts should be made to improve the working conditions of non-professional HCWs in health services.

At the time of writing, the major task for healthcare providers is to reinstate service delivery and regain programme losses without putting HCWs or patients at risk of COVID-19 infection. Doing this requires quickly integrating what we have learnt during the past year into routine service delivery. Policies have been adjusted to allow the majority of services to resume, with the exception of group-based psychosocial support and medication distribution groups. These policies now need to be analysed for sustainability, and we need to improve the integration of safer methods of service provision into health systems.

\section{Declaration. None.}

Acknowledgements. We would like to thank all the COVID and clinical coordinators for their hard work, particularly Ntsetse Kgopong, Nthabiseng Magagula, Khanyi Mthembu and Vusanimudi Tshindane, and all Anova employees for continuing to provide services over this time of adversity. Author contributions. KR and JLD conceived the analysis. SP-A, KR and JLD developed the data collection tools and procedures, and collated data. KR performed the analysis and drafted the manuscript. All authors assisted with the interpretation of the results, took part in revising the article, and gave final approval.
Funding. This study was made possible by the generous support of the American people through the US President's Emergency Plan for AIDS Relief (PEPFAR) through the United States Agency for International Development (USAID) under Cooperative Agreement number 674-A-1200015 to the Anova Health Institute. The contents are the responsibility of Anova Health Institute and do not necessarily reflect the views of USAID or the United States government. The funders had no role in study design, data collection and analysis, decision to publish, or preparation of the manuscript.

Conflicts of interest. None.

1. World Health Organization. Prevention, identification and management of health worker infection in the context of COVID-19. Interim guidance, 30 October 2020. Geneva: WHO, 2020. https://www.who.int/ publications/i/item/10665-336265 (accessed 5 November 2020).

2. Amnesty International. Global: Amnesty analysis reveals over 7,000 health workers have died from COVID-19. 3 September 2020. https://www.amnesty.org/en/latest/news/2020/09/amnesty-analysis7000-health-workers-have-died-from-covid19/ (accessed 4 November 2020).

3. Ministry of Health, Republic of South Africa. Statement on health care workers that have acquired COVID-19. 13 August 2020. https://storage.googleapis.com/stateless-bhekisisa-website/wordpressuploads/2020/08/fda38e56-hcw-13-august-2020.pdf (accessed 4 November 2020).

4. South Africa. Notice on compensation for occupationally-acquired novel corona virus disease (COVID-19) under Compensation for Occupational Injuries and Diseases Act, 130 of 1993 as amended. https://www.gov. za/sites/default/files/gcis_document/202003/43126gen 193.pdf (accessed 13 January 2021). 5. Nguyen LH, Drew DA, Graham MS, et al. Risk of COVID-19 among frontline healthcare workers and
the general community: A prospective cohort study. Lancet Public Health 2020;5(9):E475-E483. https:// doi.org/10.1016/S2468-2667(20)30164-X

6. Human Sciences Research Council. Front line talk - South African health care workers' response to the COVID-19 pandemic. Durban: HSRC, 2020. http://www.hsrc.ac.za/uploads/pageContent/12151/ FULL\%20DECK\%20OF\%20SLIDES\%20for\%20WEBINAR\%206\%20AUGUST\%202020\%20FINAL.pdf (accessed 13 January 2021).

7. Houghton C, Meskell P, Delaney H, et al. Barriers and facilitators to healthcare workers' adherence with infection prevention and control (IPC) guidelines for respiratory infectious diseases: A rapid qualitative evidence synthesis (Review). Cochrane Database Syst Rev 2020, Issue 4. Art. No. CD013582. https://doi. org/10.1002/14651858.CD013582

8. National Institute for Communicable Diseases. Clinical management of suspected or confirmed COVID-19 disease. 2020. https://www.nicd.ac.za/diseases-a-z-index/covid-19/covid-19-guidelines/ clinical-management-of-suspected-or-confirmed-covid-19-disease/ (accessed 4 November 2020).

9. National Institute for Communicable Diseases. Guidelines for symptoms monitoring and management of essential workers for COVID-19 related infection. 2020. https://www.nicd.ac.za/diseases-a-z-index/ covid-19/covid-19-guidelines/symptoms-monitoring-and-management-of-essential-workers-for-covid-
19-related-infection/ (accessed 4 November 2020). D. National Institute for Communicable Diseases. COVID-19 disease: Infection prevention and control guidelines. 2020. https://www.nicd.ac.za/diseases-a-z-index/covid-19/covid-19-guidelines/infectionrevention-and-control-guidelines/ (accessed 4 November 2020).

. Chou R, Dana T, Buckley DI, Selph S, Fu R, Totten A. Epidemiology of and risk factors for coronavirus infection in health care workers. Ann Intern Med 2020;173:120-136. https://doi.org/10.7326/M20-1632

2. Balfour T, Charalambous S, Muchiri E. An analysis of COVID-19 cases and deaths in the South African mining industry. Occup Health S Afr 2020;26(5):196-198. https://www.occhealth.co.za/?/issue/334 (accessed 13 January 2021).

13. Naidoo S, Seevnarain K, Nordstrom DL. Tuberculosis infection control in primary health clinics in eThekwini, KwaZulu-Natal, South Africa. Int J Tuberc Lung Dis 2012;16(12):1600-1604. https://doi. org/10.5588/ijtld.12.0041

14. Fennelly KP. Particle sizes of infectious aerosols: Implications for infection control. Lancet Respir Med 2020;8(9):914-924. https://doi.org/10.1016/S2213-2600(20)30323-4

15. Editorial. COVID-19 transmission - up in the air. Lancet Respir Med 2020;8(12):1159. https://doi. org/10.1016/S2213-2600(20)30514-2

16. Dai H, Zhao B. Association of the infection probability of COVID-19 with ventilation rates in confined spaces. Build Simul 2020;13:1321-1327. https://doi.org/10.1007/s12273-020-0703-5

17. Thorne C, Khozin S, McDiarmid MA. Using the hierarchy of control technologies to improve healthcare facility infection control: Lessons from severe acute respiratory syndrome. J Occup Environ Med 2004;46(7):613-622. https://doi.org/10.1097/01.jom.0000134191.92225.f2

18. Sikkema RS, Pas SD, Nieuwenhuijse DF, et al. COVID-19 in health-care workers in three hospitals in the south of the Netherlands: A cross-sectional study. Lancet Infect Dis 2020;20(11):1273-1280. https://doi. $\mathrm{org} / 10.1016 / \mathrm{S} 1473-3099(20) 30527-2$

19. Nyasulu J, Pandya H. The effects of coronavirus disease 2019 pandemic on the South African health system: A call to maintain essential health services. Afr J Prim Health Care Fam Med 2020;12(1):12-15. https://doi.org/10.4102/phcfm.v12i1.2480

20. Roberton T, Carter ED, Chou VB, et al. Early estimates of the indirect effects of the COVID-19 pandemic on maternal and child mortality in low-income and middle-income countries: A modelling study. Lancet Glob Health 2014;8(7):e901-e908. https://doi.org/10.1016//S2214-109X(20)30229-1

21. Western Cape Government: Health. Circular H 69 of 2020: Primary health care preparedness for COVID-19 epidemic in Western Cape Province. 14 May 2020. https://www.westerncape.gov.za/assets/departments/ health/h69_2020_covid-19_primary_health_care_preparedness.pdf (accessed 4 November 2020).

22. Wilkinson L, Boyles T, Moosa S, Muller M, Cooke R. COVID-19 primary care facility preparedness guide. Revised 9 July, 2020. https://differentiatedservicedelivery.org/Portals/0/adam/Content/R7yA0D-sEOFaX1908NLIw/File/COVID-19\%20Primary\%20Care\%20Facility\%20Preparedness $\% 20$ Guide $\% 20$ 09.07.2020\%20update_lowres-compressed.pdf (accessed 4 November 2020).

Accepted 11 January 2021. 